\title{
Análisis comparativo de insulina glargina frente a la insulina detemir: un modelo de minimización de costos aplicable en Colombia
}

\author{
Argemiro Fragozo', María Fernanda Puerta², Juan Diego Misas² \\ 1 Escuela de Medicina, Universidad El Bosque, Bogotá, D.C., Colombia \\ 2 Sanofi-Aventis de Colombia, S. A., Bogotá, D.C., Colombia \\ Estudio llevado a cabo en Sanofi-Aventis de Colombia, S. A.
}

Introducción. Más del $90 \%$ de los individuos diagnosticados con diabetes mellitus presentan el tipo 2 , cuya resistencia periférica a la acción de la insulina es conocida.

Objetivo. Desarrollar un modelo de minimización de costos del tratamiento con insulina glargina una vez al día o con insulina detemir, una o dos veces al día, en pacientes con diabetes mellitus de tipo 2 que requieren insulina, desde la perspectiva del tercer pagador en Colombia.

Materiales y métodos. Se hizo una búsqueda sistemática de estudios clínicos comparativos de la administración de insulina glargina e insulina detemir en pacientes con diabetes mellitus de tipo 2 que requieren insulina, con el fin de extraer los datos sobre su uso y efectividad, y sobre la frecuencia de eventos secundarios. La meta establecida de control glucémico fue de HbA1c $\leq 7 \%$. Los costos de la insulina se tomaron del Sistema Integrado de Precios de Medicamentos, 2012, del Ministerio de Salud y Protección Social, y los precios por tableta se basaron en el promedio móvil de doce meses en diciembre de 2012 según el IMS Consulting Group. Los análisis de sensibilidad se hicieron con simulaciones de Montecarlo para las dosis y los costos de la insulina.

Resultados. Cinco publicaciones cumplieron con los criterios de inclusión. El rango de la diferencia entre dosis de insulina fue de 3,2 a 33 UI. El porcentaje de pacientes que requirieron dos dosis de insulina detemir estuvo entre 12,6 y $100 \%$. No hubo diferencias significativas en los eventos hipoglucémicos. Tanto para el canal de compra al por menor como para el de compras institucionales, la diferencia de costos entre la insulin glargina y la detemir favoreció a la primera en cuatro y cinco estudios, respectivamente. Solo un estudio mostró lo contrario en lo concerniente a la venta al por menor.

Conclusiones. La diferencia en cuanto a la dosis promedio entre la insulina ganglir y la detemir, genera costos anuales que favorecen el uso de la insulina ganglir, lo que la convierte en una alternativa costo-efectiva frente a la determir.

Palabras clave: insulina/economía, costos en drogas, diabetes mellitus, hipoglucemia, monitoreo fisiológico.

doi: http://dx.doi.org/10.7705/biomedica.v35i2.2338

\section{Comparative analysis of insulin glargine vs. insulin detemir: A cost-minimization study applicable to Colombia}

Introduction: More than $90 \%$ of subjects diagnosed with diabetes mellitus present with type 2 , which is recognized for peripheral insulin resistance.

Objective: To determine the costs of achieving glycemic target with the use of basal insulin analogs, insulin glargine (IG) once a day vs. insulin detemir (ID) once or twice a day, with a cost minimization model built from a third-party payer perspective in Colombia.

Materials and methods: A systematic review of comparative clinical trials between IG and ID in patients with insulin-resistant type 2 diabetes was performed to determine data of use, effectiveness and frequency of and adverse events. The goal of glycemic control (effectiveness measure) was defined as $\mathrm{HbA} 1 \mathrm{c} \leq 7 \%$. The costs of insulin were extracted from the Integrated System of Medication Prices 2012 (Ministerio de Salud y Protección Social de Colombia) and the IMS Consulting Group mobile average cost for the past year as of December, 2012. Sensitivity analyses were performed via Montecarlo simulations for dose and medication costs (insulin).

\footnotetext{
Contribución de los autores:

Argemiro Fragozo: desarrollo de la idea y revisión de la literatura

María Fernanda Puerta: análisis de la información

Juan Diego Misas: desarrollo de la idea, diseño del estudio, estimación de costos y análisis farmacoeconómico

Todos los autores participaron en la escritura del manuscrito.
} 
Results: Five publications met inclusion criteria. The range of the difference between insulin doses was 3.2 IU to 33 IU. The percentage of patients requiring two ID doses was $12.6-100 \%$. There were no significant differences in hypoglycemic events. For both retail and institutional channels, there was a higher differential cost between IG vs. ID favoring IG in 4 and 5 studies, respectively. For the retail channel only one study showed the opposite results.

Conclusions: As only medication costs are considered, differences in insulin units between IG and ID result in a differential cost in favor of IG that makes it a cost/effective alternative.

Key words: Insulin/economics, drug cost, diabetes mellitus, hypoglycemia; monitoring, physiologic. doi: http://dx.doi.org/10.7705/biomedica.v35i2.2338

La diabetes mellitus agrupa una serie de enfermedades metabólicas que afectan a millones de personas en todo el mundo. Se calcula que su prevalencia para el 2011 fue de 366 millones de casos $(8,3 \%$ de la población mundial) y se prevé una prevalencia de 552 millones de pacientes $(9,9 \%)$ para el año 2030 (1). Más del $90 \%$ de los individuos diagnosticados con diabetes presentan diabetes mellitus de tipo 2. Esta forma de diabetes mellitus se reconoce por su resistencia periférica a la acción de la insulina y por una disfunción progresiva de la célula $\beta$, por lo cual el tratamiento de reemplazo con insulina a menudo es necesario (2).

Con el uso de la insulina se busca alcanzar un perfil glucémico cercano al que tendría un paciente sano, con el menor riesgo de hipoglucemia y la menor ganancia de peso. Aunque el tratamiento óptimo con insulina en la diabetes mellitus de tipo 2, está sujeto a controversias, el tratamiento de reemplazo suele iniciarse con insulina basal, bien sea sola o conjuntamente con agentes orales (3).

Actualmente, se dispone de insulinas humanas de acción intermedia, como la insulina de protamina neutra Hagedorn (Neutral Protamine Hagedorn, $\mathrm{NPH}$ ), o de insulinas análogas basales, como la insulina glargina (Lantus ${ }^{\circledR}$ de Sanofi, Francia) y la insulina detemir (Levemir ${ }^{\circledR}$ de Novo Nordisk A/S, Dinamarca) (4,5). Estas dos últimas ofrecen una eficacia similar a la insulina NPH, pero permiten esquemas de tratamiento más flexibles y simples, con menor riesgo de hipoglucemia, lo cual es una característica importante a la hora de tomar la decisión de iniciar la administración de insulina de manera oportuna (6).

\footnotetext{
Correspondencia:

Juan Diego Misas, Departamento Médico, Sanofi-Aventis de Colombia, S. A., Transversal 23 N 97-73, piso 9, Bogotá, D.C., Colombia

Teléfono: (571) 593 6039; fax: (571) 7444237

diego.misas@sanofi.com

Recibido: 17/03/14; aceptado: 28/01/15
}

Las diferencias en el perfil farmacocinético de la insulina glargina y de la detemir pueden tener un impacto económico relevante (7). Según las instrucciones del fabricante y con dichos perfiles farmacocinéticos, se recomienda que la primera se administre una sola vez al día y, la segunda, una o dos veces al día $(4,5)$. Lo anterior puede traducirse en diferencias en el número de unidades de análogos de insulina necesario para lograr las mismas metas de HbA1c y la cantidad de insumos requeridos para su administración, lo que resulta relevante en el momento de elegir uno de los análogos de la insulina, dado su impacto presupuestal desde la perspectiva del pagador. Por lo tanto, es necesario cuantificar los costos económicos en los que se incurre para alcanzar metas glucémicas similares con cada tipo de insulina análoga. Hasta la fecha, en Colombia no se ha hecho un análisis de esta naturaleza.

En este estudio se desarrolló un modelo de minimización de costos para estimar los costos anuales de pacientes con diabetes mellitus de tipo 2 que requieren insulina y siguen un esquema de tratamiento con insulina glargina una vez al día o insulina detemir, una o dos veces al día, desde la perspectiva del tercer pagador. El análisis de minimización de costos supone que la eficacia, la efectividad y la seguridad de las opciones de atención en salud contempladas sean las mismas (8). Por lo tanto, la única diferencia entre las opciones terapéuticas fue la de su costo, lo que permitió saber cuál de las alternativas comparadas generaba menos costos para el pagador.

\section{Materiales y métodos}

Se hizo una búsqueda en Pubmed (http://www.ncbi. nlm.nih.gov/pubmed/) de estudios comparativos de insulina glargina e insulina detemir para pacientes con diabetes mellitus de tipo 2 que requerían insulina, ajustada a los siguientes criterios de inclusión: estudios clínicos observacionales o experimentales, con información sobre la dosis de insulina, la frecuencia de aplicación, la hemoglobina 
'glucosilada', los eventos hipoglucémicos y la ganancia de peso; se utilizaron los términos MeSH [insulin analogues] AND [insulin glargine] AND [insulin detemir] AND [glargine] AND [detemir] AND [type 2 diabetes] AND [Diabetes] AND [Effectiveness] AND [Glycaemia] AND [HbA1c].

Los límites de la búsqueda fueron los siguientes: abstract available, published since 2000, human clinical trials, meta-analysis, randomized controlled trial, and disease registry. Además, se buscaron en Pubmed datos con base en los siguientes términos de búsqueda no contemplados en los términos MeSH: Comparison of insulin detemir AND insulin glargine. La búsqueda de literatura se hizo a partir del año 2000, dado que la insulina glargina estuvo disponible en el mercado a partir del 2001 y, la insulina detemir, del 2004 (4,5).

En cada estudio seleccionado se identificó la dosis media y la desviación estándar en cada una de los grupos de tratamiento por tipo de análogo de insulina (glargina y detemir). Igualmente, se extrajo la información relacionada con el porcentaje de eventos hipoglucémicos en los pacientes tratados con glargina y detemir y el porcentaje de aquellos tratados con insulina detemir que requirieron dos dosis para alcanzar el control glucémico.

Los costos de las insulinas se tomaron de la Circular 07 de 2013 expedida por la Comisión Nacional de Precios de Medicamentos y Dispositivos Médicos del Ministerio de Salud y Protección Social de Colombia, en la cual se regula el precio por unidad internacional de los análogos de insulina con destino a las instituciones de salud. En cuanto a los precios al por menor, se tomó la información de los precios por tableta, resultado del promedio móvil de doce meses con corte a diciembre de 2012 calculado por el IMS Consulting Group (9). El costo de la unidad internacional de insulina glargina y de insulina detemir para suministro a instituciones, es de COP\$ 83,59 y COP\$ 87,97, respectivamente, y de COP\$ 169 y COP\$159,7 para suministro al por menor. Cabe mencionar que a partir del 2013 la regulación internacional de precios aplica en los dos ámbitos, siendo un punto de referencia en el suministro al por menor. Los análisis de sensibilidad se hicieron con simulaciones de Montecarlo tanto en lo referente a las dosis como a los precios de las insulinas, asumiendo una distribución normal en los procesos de generación de números aleatorios (10). Se construyeron los análisis de cuadrante, así como las curvas de aceptabilidad, con el objetivo de determinar la proporción de pacientes que representó mayores costos y requirió mayores dosis de alguno de los dos análogos de insulina (11). Los costos en pesos corrientes colombianos corresponden a valores de 2013.

Para la estimación de los costos de los análogos de insulina para el manejo del paciente diabético, se tomaron las dosis medias de insulina glargina e insulina detemer en cada uno de los estudios seleccionados y se multiplicaron por el precio calculado para el suministro al por menor y por el precio regulado de referencia para el ámbito institucional. De igual forma, con base en los hallazgos de los estudios seleccionados en lo relativo a la desviación estándar de la dosis promedio, se multiplicó por los precios ya mencionados para cada canal de comercialización.

\section{Resultados}

\section{Búsqueda sistemática de publicaciones y extracción de datos}

La búsqueda sistemática de publicaciones de estudios clínicos comparativos del uso de la insulina glargina y la detemir con términos MeSH, en pacientes con diabetes mellitus de tipo 2 que requirieran insulina, arrojó 41 publicaciones. De estas, se seleccionaron cuatro luego de la revisión crítica de sus resúmenes, dado que se trataba de comparaciones 'cabeza a cabeza' de las dos moléculas, se reportaba la dosis de insulina, la duración del tratamiento y la meta de hemoglobina 'glucosilada'. En una segunda búsqueda con base en términos diferentes a los $\mathrm{MeSH}$, se encontraron 40 publicaciones, de las cuales se seleccionó solamente una. Se descartaron 38 artículos en razón de su alcance y uno por estar repetido. En total, entre los resultados de las búsquedas mencionadas se seleccionaron cinco publicaciones (12-16), cuyo texto completo fue consultado. Las características operativas y los datos extraídos de los estudios seleccionados se describen en el cuadro 1.

Como se aprecia en dicho cuadro, los estudios seleccionados fueron publicados entre 2007 y 2010. Cuatro de los estudios son multicéntricos, multinacionales, abiertos, prospectivos y de grupos paralelos. Uno de los estudios es un análisis retrospectivo de un registro de enfermedad desarrollado por Currie, et al. (16), en el Reino Unido. Cabe destacar que en el manuscrito original de este estudio no se describen las dosis promedio por paciente, por lo que fue necesario utilizar la información descrita en el estudio de Poole, et al. (17), en el que se estimaron las dosis promedio 
por año para pacientes tratados con glargina y con detemir, información esta proveniente del registro en cuestión.

Existen variaciones en el número de semanas de seguimiento de los participantes en los estudios prospectivos. Específicamente, Rosenstock, et al. (12), y Hollander, et al. (13), hicieron un seguimiento durante 52 semanas; Swinnen, et al. (16), hicieron un seguimiento de 24 semanas, en tanto que Raskin, et al. (17), lo hicieron durante 26 semanas.

Los pacientes tratados con insulina glargina una sola vez al día, como también los tratados con insulina detemir con una o dos dosis diarias, obtuvieron reducciones similares en el nivel de $\mathrm{HbA1c}$ y en el examen de glucemia en ayunas al final del tratamiento. Sin embargo, en el estudio de Rosenstock, et al, (14), $55 \%$ de los pacientes requirió aplicación de insulina detemir dos veces al día para alcanzar el mismo control glucémico logrado con la insulina glargina una vez al día, y en el estudio de Hollander, et al. (15), sucedió igual en el $57 \%$ de los casos. De manera similar, no se reportaron diferencias significativas entre los brazos de comparación en ninguno de los estudios incluidos en el presente análisis, en cuanto al porcentaje de eventos hipoglucémicos por paciente y por año. Sin embargo, en el estudio de Raskin, et al. (17), se evidenció una mayor frecuencia de eventos hipoglucémicos en ambos grupos, lo que pudo deberse a las dosis de insulina empleadas en el estudio. En conclusión, la insulina glargina y la insulina detemir tuvieron la capacidad de lograr disminuciones comparables de $\mathrm{HbA} 1 \mathrm{c}$ en los estudios seleccionados, aunque con diferencias en el número de unidades necesarias para lograr el mismo control glucémico.

\section{Estimación de costos}

La estimación de costos anuales tomando como referencia los resultados de los estudios seleccionados, se describe en el cuadro 2. El análisis de los costos de los cuatro estudios permite demostrar que los precios al por menor de la insulina glargina eran más ventajosos que los de la insulina detemir. Tan solo en el análisis derivado de los resultados del estudio de Raskin, et al. (17), se observó una ventaja de la insulina detemir frente a la insulina glargina, aunque no significativa. Al examinar cada estudio específico, en el análisis de los costos derivado de los resultados del estudio desarrollado por Rosenstock, et al. (14), se observó que el costo anual del tratamiento con insulina glargina era COP\$ 803.229 más económico que el tratamiento con insulina detemir. En el análisis de costos de los datos del estudio de Hollander, et al. (15), esta diferencia aumentó, pues el tratamiento con insulina detemir costaba COP\$984.014 más que el tratamiento con insulina glargina. En el caso de los costos derivados de los resultados del estudio de Swinnen, et al. (16), la diferencia entre los tratamientos fue de COP $\$ 1^{\prime} 775.211$; sin embargo, la desviación estándar de dichos costos es lo suficientemente amplia como para que esa ventaja comparativa pueda no ser significativa (cuadro 2). Además, cabe destacar que existe una gran variabilidad en los costos calculados que se deriva de la amplia desviación estándar de la dosis por día reportada en el estudio de Raskin, et al. (17).

En el caso de las instituciones, los análisis de costos anuales mostraron resultados similares a los encontrados en la distribución al por menor, lo que ratifica que el tratamiento con insulina glargina fue el más económico. Debe destacarse que el mayor ahorro y la menor desviación estándar se encontraron en los costos derivados de los resultados del estudio de Hollander, et al. (15), en el cual se evidenció una diferencia de costos de COP\$ 656.712 a favor de la insulina glargina frente a la insulina detemir. Además, en los análisis de costos basados en los resultados de los estudios de Rosenstock, et al. (14), Hollander, et al. (13), y Currie, et al. (16), la diferencia de costos a favor de la insulina glargina fue de proporciones similares. En el caso del análisis de costos de los resultados del estudio de Raskin, et al. (15), la diferencia de costo fue a favor de la insulina detemir, pero esta no fue significativa dada la elevada desviación estándar (cuadro 2).

\section{Análisis de sensibilidad}

Dada la variabilidad de las dosis promedio de insulina por día y de los costos de tratamiento, fue necesario hacer un análisis de sensibilidad en el que se introdujeron cambios en las estimaciones promedio y se evaluó la solidez del análisis frente a dichos cambios (10). Para cada uno de los estudios se hicieron análisis de Montecarlo con 1.000 simulaciones, con una distribución normal en la generación de los números aleatorios y los valores promedio y la desviación estándar en dosis ya explicados.

En el caso de los precios al por menor, se tomó el promedio reportado por el IMS Consulting Group, y la desviación estándar se calculó como la mitad del valor entre el mínimo y el máximo reportado. En el caso de los precios para instituciones, dado que son 

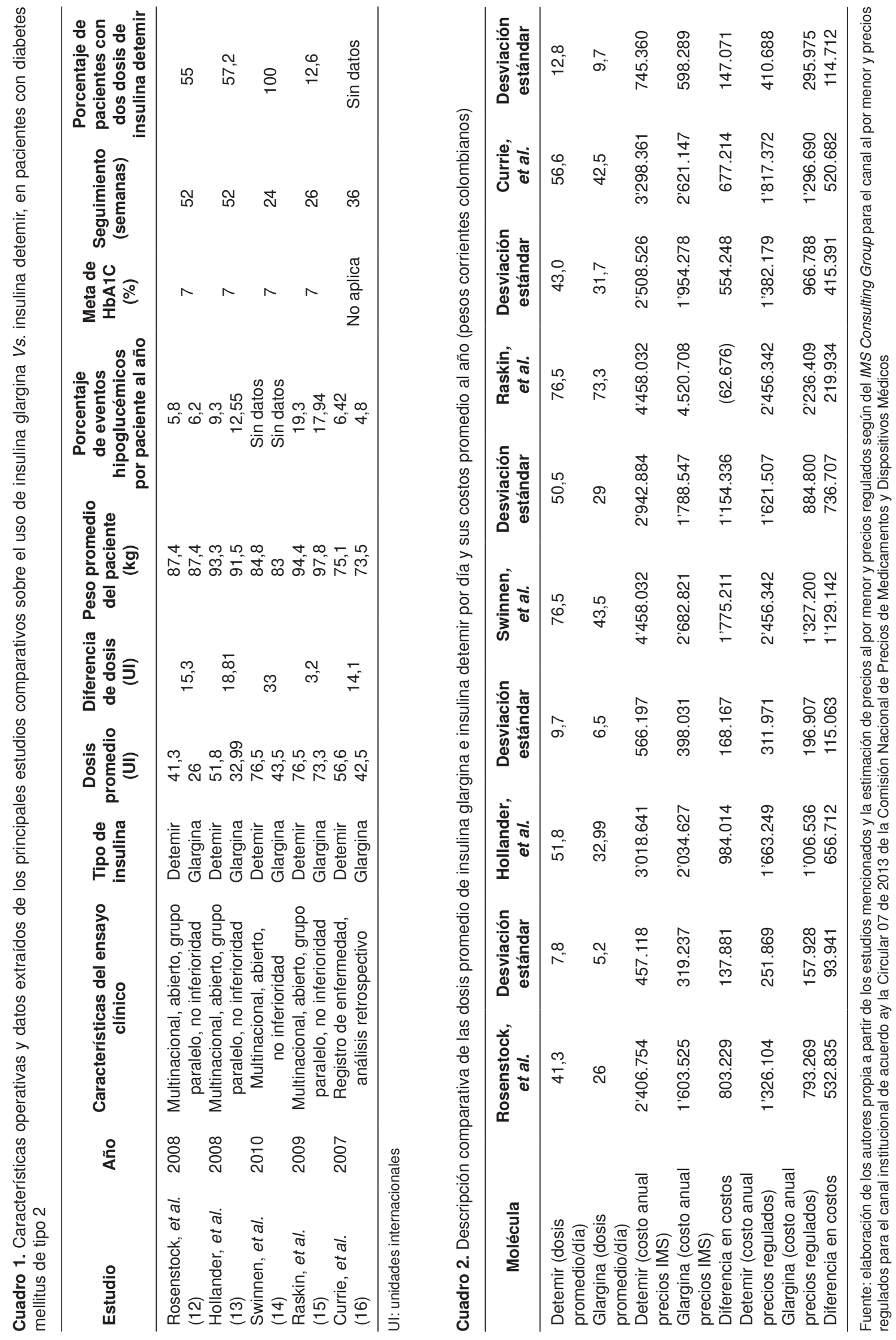
regulados no habría desviaciones estándar, ya que se tendría un único precio para cada tipo de insulina. Sin embargo, para efectos de las simulaciones se calculó un margen de intermediación de 5 a $10 \%$, lo que permitió construir las desviaciones estándar para los precios de cada insulina (cuadro 2).

En la figura 1, A-E, el punto de referencia $(0,0)$ corresponde a la insulina glargina, y la nube de puntos representa las simulaciones de las diferencias en las dosis y los costos frente a la insulina detemir. En el caso de la figura $1 \mathrm{~A}$, derivada de los resultados de Rosenstock, et al. (12), la mayoría de las simulaciones demostraron que los pacientes tratados con insulina detemir requirieron un mayor número de dosis y que el costo anual del tratamiento fue mayor que con la insulina glargina
(96\% de los pacientes requirió más dosis y en $93,8 \%$ de los casos los costos fueron mayores). La figura 1B, derivada de los resultados de Hollander, et al. (13), presenta la misma tendencia de las estimaciones hechas en el modelo derivado de los resultados de Rosenstock, et al. (12), y casi todas las simulaciones (pacientes) demostraron que con la insulina detemir se requería un mayor número de dosis y que los costos anuales del tratamiento eran mayores (98\% de los pacientes requirieron más dosis y en $90 \%$ de los casos el costo fue mayor). La figura $1 \mathrm{C}$, derivada de los resultados de Swinnen, et al. (14), demuestra que el $70 \%$ de los pacientes tratados con insulina detemir requirieron más dosis y en $65 \%$ de los casos se incurrió en un gasto mayor. La figura 1D, derivada de los resultados
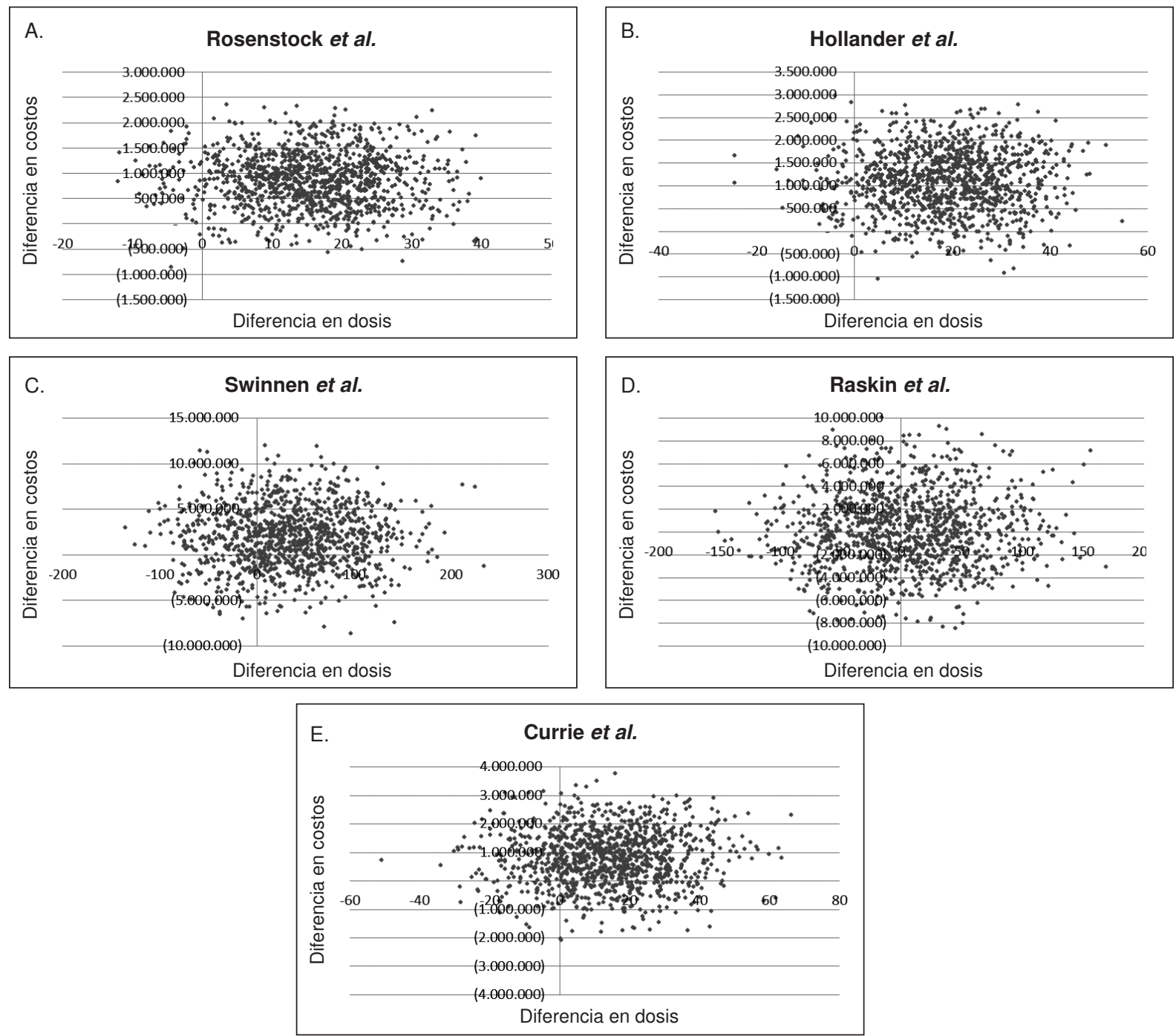

Figura 1. Análisis de sensibilidad por diferencia de dosis y diferencia en costos (IMS). Diferencia en dosis: unidades internacionales de insulina; diferencia de costos: pesos corrientes colombianos 
de Raskin, et al. (15), muestra que, dada la alta desviación estándar en la dosis, la dispersión en los datos fue mayor, lo que significó que 52,2\% de los pacientes requiriera más dosis y en $48 \%$ de los casos el costo fuera mayor. Cabe anotar que este es el único estudio en el cual no se observó una tendencia clara a favor o en contra de una u otra de las insulinas. Por último, la figura 1D, derivada de los resultados de Currie, et al. (16), se demuestra que el $78 \%$ de los pacientes tratados con detemir requirieron más dosis y en $75 \%$ de los casos los costos de manejo fueron más elevados.

\section{Discusión}

La prevalencia de diabetes mellitus ha aumentado rápidamente en años recientes. El envejecimiento de la población mundial, los malos hábitos alimentarios, el sedentarismo y la obesidad parecen ser las causas más frecuentemente identificadas en los estudios epidemiológicos (1). Sin duda, la diabetes mellitus se ha convertido en una pandemia y en un problema de salud pública tanto por sus elevadas tasas de morbimortalidad, como por el alto costo que su tratamiento representa para los sistemas de salud, ya que los pacientes diabéticos consumen de dos a cinco veces más recursos sanitarios que la población no diabética (18-20). De ahí la necesidad de llevar a cabo estudios presupuestales aplicados a la realidad de nuestros países. En Colombia carecemos de este tipo de análisis.

En la presente investigación, el análisis de costos basado en los resultados clínicos de estudios comparativos en pacientes con diabetes mellitus de tipo 2, demostró que aquellos tratados con insulina glargina consumían anualmente menos recursos económicos que los tratados con insulina detemir. En el análisis de costos basado en los precios al por menor y aplicado a los resultados de cuatro de los cinco estudios (12-16), se evidenció una ventaja comparativa para la insulina glargina. Solo en el caso del análisis de costos derivado de los resultados del estudio de Raskin, et al. (15), la diferencia estuvo a favor de la insulina detemir, aunque dicha diferencia no fue significativa debido a la alta desviación estándar. En cuanto al ámbito de las instituciones, los resultados del análisis de costos fueron similares a los del análisis basado en los precios al por menor; de nuevo aquí la diferencia a favor de la insulina detemir solo se presentó en el estudio de Raskin, et al. (15), pero debido a la elevada desviación estándar mencionada, los resultados no fueron coherentes.
Al multiplicar las diferencias de costo del tratamiento anual con cada una de las insulinas disponibles en el país por el número de pacientes con diabetes de tipo 2 que requieren análogos de insulina (alrededor de 110.000 pacientes con base en una prevalencia del $5 \%$ para Colombia, de los cuales el $4 \%$ son tratados con análogos de insulina) (21), se calculó un posible ahorro que oscila entre COP $\$ 24.000$ millones y COP\$ 124.000 millones de pesos al año en el caso de las instituciones.

Es importante resaltar que el análisis se basó en el supuesto de que la eficacia de los dos análogos de insulina es la misma y que con cualquiera de las dos se logra el mismo control glucémico en esta población. Sin embargo, el hecho de que se requiera un mayor número de unidades internacionales de insulina detemir que de insulina glargina al día por paciente, con el consiguiente aumento de los insumos de aplicación, es un factor que puede incrementar el costo del tratamiento. Los análisis de sensibilidad permitieron corroborar que las variaciones en el precio de ambos medicamentos y en las dosis promedio requeridas para alcanzar un mismo nivel de control glucémico, no alteran el hecho de que los costos del tratamiento favorezcan el uso de la insulina glargina.

Los resultados de este análisis están limitados por diversos hechos. La información de referencia utilizada para determinar la eficacia clínica se deriva de estudios foráneos que probablemente no reflejan la realidad del país. Así pues, se requieren estudios en la población local para corroborar nuestros hallazgos. Además, los resultados del presente análisis pueden variar con la inclusión de nuevos estudios clínicos con similares características operativas y no son extrapolables a estudios realizados con moléculas con características biológicas similares, que usualmente tienen un menor precio en el mercado y sobre las cuales no hay estudios 'cabeza a cabeza' de eficacia y seguridad. Sumado a lo anterior, debe tenerse en cuenta que este estudio solo consideró la diferencia en el costo del uso de insulinas. Cabe mencionar que el hecho de que una proporción significativa de pacientes con insulina detemir reciba dos dosis al día incrementa los costos en insumos médicos tales como agujas y tirillas, lo que aumenta el ahorro obtenido con el uso de la insulina glargina más allá de lo señalado en este estudio.

La evaluación de alternativas de tratamiento que permitan el control glucémico de los pacientes al menor costo económico posible, es valiosa y 
necesaria para un sistema de salud con recursos financieros limitados.

La diferencia en la dosis promedio entre la insulina glargina y la insulina detemir tiene una incidencia significativa en los costos anuales que favorece el uso de la primera, pues es una alternativa más costo-efectiva.

\section{Agradecimientos}

A Dimas Felipe Herrera y Luis Felipe Orozco, por la revisión y los comentarios sobre el manuscrito.

\section{Conflicto de intereses}

Argemiro Fragozo ha recibido patrocinio para la asistencia a actividades de educación médica continua de parte de Sanofi, Novo, así como honorarios de Sanofi por concepto de investigación y consultorías. María Fernanda Puerta y Juan Diego Misas son empleados de Sanofi-Aventis de Colombia, S. A.

\section{Financiación}

Sanofi-Aventis de Colombia, S. A.

\section{Referencias}

1. International Diabetes Federation. IDF Diabetes Atlas. Fecha de consulta: 16 de noviembre de 2012. Disponible en: http://www.idf.org/diabetesatlas/5e/es/la-carga-mundial ?language $=\mathrm{es}$.

2. American Diabetes Association. Standards of medical care in diabetes-2013. Diabetes Care. 2013;36(Suppl.1):S11-66. http://dx.doi.org/10.2337/dc13-S011

3. Leahy, JL. Insulin therapy in type 2 diabetes mellitus. Endocrinol Metab Clin North Am. 2012;41:119-44. http:// dx.doi.org/10.1016/j.ecl.2012.03.004

4. Sanofi-Aventis de Colombia, S. A. Información para prescribir Lantus ${ }^{\circledR}$. Bogotá: Sanofi-Aventis de Colombia, S. A; 2003.

5. Novo Nordisk. Información para prescribir Levemir ${ }^{\circledR}$ Buenos Aires: Novo Nordisk; 2009.

6. Little S, Shaw J, Home P. Hypoglycemia rates with basal insulin analogs. Diabetes Technol Ther. 2011;13 (Suppl.1):S53-64. http://dx.doi.org/10.1089/dia.2011.0022

7. Porcellati F, Rossetti P, Busciantella NR, Marzotti S, Lucidi P, Luzio, et al. Comparison of pharmacokinetics and dynamics of the long-acting insulin analogs glargine and detemir at steady state in type 1 diabetes. Diabetes Care. 2007;30:2447-52. http://dx.doi.org/10.2337/dc07-0002

8. Drummond $\mathbf{M}$, Sculpher $\mathbf{M}$, Torrnace $\mathbf{G}, \mathbf{O}^{\prime}$ Brian $\mathbf{B}$, Stoddart G. Methods for the economic evaluation of health care programmes. Third edition. Oxford: Oxford University Press; 2005.

9. Long D. The US Pharmaceutical Market: Trends, Issues, \& Outlook. Bogotá: IMS Consulting Group; 2011.
10. Fishman GS. Monte Carlo: Concepts, algorithms, and applications. First edition. New York: Springer-Verlag; 1995.

11. Fenwick E, Marshall D, Levy A, Nichol G. Using and interpreting cost-effectiveness acceptability curves: An example using data from a trial of management strategies for atrial fibrillation. BMC Health Serv Res. 2006,6:52. http:// dx.doi.org/10.1186/1472-6963-6-52

12. Rosenstock J, Davies M, Home P, Larsen J, Koenen C, Schernthaner G. A randomised, 52-week, treat-totarget trial comparing insulin detemir with insulin glargine when administered as add-on to glucose-lowering drugs in insulin-naive people with type 2 diabetes. Diabetologia. 2008;51:408-16. http://dx.doi.org/10.1007/s00125-007-0911-x

13. Hollander P, Cooper J, Bregnhoj J, Bang-Perdensen C. A 52-week, multinational, open-label, parallel-group, non-inferiority, treat-to-target trial comparing insulin detemir with insulin glargine in a basal-bolus regimen with mealtime insulin aspart in patients with type 2 diabetes. Clin Ther. 2008;30:1976-87. http://dx.doi.org/10.1016/j. clinthera.2008.11.001

14. Swinnen S, Dain MP, Aronson R, Davies M, Gerstein HC, Pfeiffer AF, et al. A 24-week, randomized, treat-to-target trial comparing initiation of insulin glargine once-daily with insulin detemir twice-daily in patients with type 2 diabetes inadequately controlled on oral glucose-lowering drugs. Diabetes Care. 2010;33:1176-8. http://dx.doi.org/10.2337/ dc09-2294

15. Raskin P, Gylvin T, Weng W, Chaykin L. Comparison of insulin detemir and insulin glargine using a basal-bolus regimen in a randomized, controlled clinical study in patients with type 2 diabetes. Diabetes Metab Res Rev. 2009;25:542-8. http://dx.doi.org/10.1002/dmrr.989

16. Currie C, Pool C, Tetlow T, Holmes P, McEwan P. The outcome of care in people with type 1 and type 2 diabetes following switching to treatment with either insulin glargine or insulin detemir in routine general practice in the UK: A retrospective database analysis. Adv Ther. 2011;28:100011. http://dx.doi.org/10.1007/s12325-011-0074-5

17. Poole C, Tedlow T, Ewan P, Holmes P, Curie C. The prescription cost of managing people with type 1 and type 2 diabetes following initiation of treatment with either insulin glargine or insulin detemir in routine general practice in the UK: A retrospective database analysis. Curr Medical Res Opin. 2007;23 (Suppl.1):41-8.

18. Hart W, Collazo-Herrera M. Costos del diagnóstico y tratamiento de la diabetes mellitus en diferentes países del mundo. Rev Cubana Endocrinol. 1998;9:212-20.

19. Barceló A, Aedo C, Rajpathak S, Robles S. The cost of diabetes in Latin America and the Caribbean. Bull World Health Organ. 2003;81:19-27. http://dx.doi.org/10.1590/ S0042-96862003000100006

20. Hogan P, Dall T, Nikolov P, American Diabetes Association. Economic costs of diabetes in the US in 2002. Diabetes Care. 2003;26:917-32. http://dx.doi.org/10.2337/ diacare.26.3.917

21. Cegedim. Diabetrack- Estudio de seguimiento de diabetes, 2012. Bogotá: Cegedim; 2012. 